\title{
Vivax malaria in pregnancy and lactation: a long way to health equity
}

\author{
Tobias Brummaier ${ }^{1,2,3,4^{*}+} \oplus$, Mary Ellen Gilder ${ }^{1 \dagger}$, Gornpan Gornsawun ${ }^{1}$, Cindy S. Chu ${ }^{1,4}$, Germana Bancone ${ }^{1,4}$, \\ Mupawjay Pimanpanarak ${ }^{1}$, Kesinee Chotivanich ${ }^{5}$, François Nosten ${ }^{1,4}$ and Rose McGready ${ }^{1,4}$
}

\begin{abstract}
Background: The Sustainable Development Goals (SDG) call for increased gender equity and reduction in malariarelated mortality and morbidity. Plasmodium vivax infections in pregnancy are associated with maternal anaemia and increased adverse perinatal outcomes. Providing radical cure for women with 8-aminoquinolines (e.g., primaquine) is hindered by gender-specific complexities.
\end{abstract}

Case presentation: A symptomatic episode of vivax malaria at 18 weeks of gestation in a primigravid woman was associated with maternal anaemia, a recurrent asymptomatic $P$. vivax episode, severe intra-uterine growth restriction with no other identifiable cause and induction to reduce the risk of stillbirth. At 5 months postpartum a qualitative glucose-6-phosphate dehydrogenase (G6PD) point-of-care test was normal and radical cure with primaquine was prescribed to the mother. A 33\% fractional decrease in haematocrit on day 7 of primaquine led to further testing which showed intermediate phenotypic G6PD activity; the G6PD genotype could not be identified. Her infant daughter was well throughout maternal treatment and found to be heterozygous for Mahidol variant.

Conclusion: Adverse effects of vivax malaria in pregnancy, ineligibility of radical cure for pregnant and postpartum women, and difficulties in diagnosing intermediate levels of G6PD activity multiplied morbidity in this woman. Steps towards meeting the SDG include prevention of malaria in pregnancy, reducing unnecessary exclusion of women from radical cure, and accessible quantitative G6PD screening in P. vivax-endemic settings.

Keywords: Plasmodium vivax, Equity, Primaquine, Radical cure, G6PD deficiency

\section{Background}

The Sustainable Development Goals (SDG) highlight freedom from the adverse effects of malaria (SDG 3.3) and gender equity (SDG 4) as global priorities for this decade. The intersection between health and gender equity has been recognized, and gender-specific inequities in the treatment and prevention of malaria have been a focus of recent quantitative and qualitative research [1]. Gender equity in health care requires not only equal

\footnotetext{
*Correspondence: tobias.brummaier@gmx.at

${ }^{\dagger}$ Tobias Brummaier and Mary Ellen Gilder contributed equally to this work

1 Shoklo Malaria Research Unit, Mahidol-Oxford Tropical Medicine Research Unit, Faculty of Tropical Medicine, Mahidol University, P.O. Box 46, 68/31 Bann Tung Road, Mae Sot 63110, Thailand

Full list of author information is available at the end of the article
}

access to health services, but also unequal access in response to unequal burden. In other words, one of the principles of gender equity requires that services for women not simply mirror services for men but differ from services for men in situations where women's needs or disease processes differ [2].

The World Health Organization (WHO) estimated the global incidence of Plasmodium vivax to be 7.5 million cases in 2017, and it is the most prevalent malaria species in Southeast Asia [3]. Women and their fetuses uniquely suffer from the complications of malaria in pregnancy $[4$, 5], including those associated with P. vivax infection [6].

Plasmodium vivax and Plasmodium ovale are the only two malaria species capable of relapses, due to the presence and subsequent activation of dormant liver stages 
known as hypnozoites. Treatment of the blood stages of acute vivax malaria relies on the schizontocidal agent chloroquine (CHQ) in most parts of the world, and is safe in pregnancy [7]. Relapse prevention is achieved with the 8-aminoquinolines primaquine (PMQ) or tafenoquine (TQ), both active against $P$. vivax hypnozoites $[7,8]$ and contra-indicated in pregnancy as the glucose-6-phosphate dehydrogenase (G6PD) status of the fetus cannot be determined antenatally in most malaria endemic settings.

The use of PMQ for radical cure is complicated by a few factors, including adherence to a 14-day course, commonly observed side effects such as abdominal pain and, specifically in G6PD-deficient individuals, the risk of drug-induced haemolysis [9-12]. TQ provides radical cure in a single dose, improving adherence, but has not yet been implemented widely since receiving approval from the US Food and Drug Administration (FDA) in 2018 [13]. A major barrier to TQ roll-out is the poor sensitivity of common qualitative point-of-care tests to detect intermediate levels of G6PD deficiency in heterozygote females who are at risk for haemolysis.

G6PD deficiency is caused by mutations of the G6PD gene, which is located on the $\mathrm{X}$-chromosome; genotypes and phenotypic expression are different in males and females. Males are either hemizygous wild types (with a normal phenotype) or hemizygous mutated (with a deficient phenotype), and females can be homozygous mutated (with a deficient phenotype), homozygous wild type (with a normal phenotype) or heterozygous. The G6PD enzymatic activity of heterozygous females includes a spectrum of activity from partial deficiency to normal [14]. With qualitative G6PD rapid tests, patients can only be classified as deficient or normal according to the test threshold $[15,16]$. As a result, heterozygous females with intermediate enzymatic activity are usually diagnosed as G6PD normal even though they are susceptible to PMQ- and TQ-induced haemolysis [17]. If qualitative G6PD tests are done in the presence of anaemia or a haemolytic episode, false normal results are more likely, especially for heterozygote females [18]. Some point-ofcare quantitative G6PD tests have now been validated in laboratory settings $[19,20]$ but field validation for use in malaria case management has not yet been completed.

As there are no safe options for radical cure during pregnancy, the WHO conditionally recommends radical cure postpartum, after the infant has reached 6 months of age $[7,21]$. On the Thailand-Myanmar border the strongest risk factor for $P$. vivax in the first 12 weeks postpartum was a history of $P$. vivax infection during the previous 9 months [22]. Exclusion of pregnant and lactating females from radical cure potentially affects about $13 \%$ of females in malaria-endemic areas [6]. Providing PMQ postpartum for radical cure of $P$. vivax is essential in Southeast Asia because the majority of $P$. vivax infections are attributed to relapse [23, 24]. In the absence of timely radical cure, relapses may cause illnesses and anaemia in the postpartum period, and deleterious effects in a subsequent pregnancy, increasing the risk of maternal morbidity (mostly due to anaemia), fetal loss (due to miscarriage and stillbirth) and neonatal mortality (due to pre-term birth, intra-uterine growth restriction (IUGR) and low birth weight) $[4,5,25]$.

The aim of reporting this case is to describe the multiple gender-specific morbidities suffered by a woman infected with $P$. vivax during pregnancy and to discuss potential research and programmatic priorities that could improve gender equity in $P$. vivax prevention and treatment.

\section{The case}

History and examination

An 18-year-old, primigravid, pregnant Burman woman with an unremarkable medical history, presented to the antenatal care (ANC) clinic of Shoklo Malaria Research Unit (SMRU) on the Thailand-Myanmar border. She reported a 7-day history of fever, chills, rigour, headache, dizziness, occasional palpitations, general weakness as well as muscle and joint pain. She used an impregnated bed net nightly and denied having any previous malaria infection. Obstetric ultrasound examination confirmed a viable fetus with an estimated gestational age (EGA) of 18 weeks and 4 days. The peripheral blood smear was positive for $P$. vivax (Table 1).

\section{Laboratory findings}

Haematologic laboratory parameters on admission showed a haematocrit (HCT) of $31.2 \%$ and haemoglobin of $10 \mathrm{~g} / \mathrm{dL}$. The reticulocyte count was elevated at 54 per 1000 red blood cells (normal range 8-20) indicating increased erythropoiesis. Haemoglobin typing, G6PD fluorescent spot test (FST), oral glucose tolerance test were normal and VRDL, HIV and urine culture were negative.

\section{Treatment}

The patient consented to participate in a randomized, controlled, treatment trial (ClinicalTrials.gov Identifier NCT01054248) and received an augmented regimen of artemether-lumefantrine $(\mathrm{COA}+)$ with 5 tablets twice daily for 4 days (each dose contained $100 \mathrm{mg} / 600 \mathrm{mg}$ artemether/lumefantrine). As per WHO recommendations for pregnant women, PMQ was not given. Chloroquine chemoprophylaxis was not given 
Table 1 Vital signs and blood profiles at the first and second Plasmodium vivax episode

\begin{tabular}{lll}
\hline & 1st episode & 2nd episode \\
\hline Schizontocidal treatment & COA+ & CHQ \\
EGA (weeks + days) & $18+4$ & $35+4$ \\
Maternal vital signs & & \\
Heart rate (beats per minute) & 70 & 92 \\
Blood pressure (mmHg) & $110 / 60$ & $120 / 80$ \\
Respiratory rate (per minute) & 24 & 23 \\
Body temperature (degree celsius) & 38.8 & 36.1 \\
Weight (kg) & 57 & 66 \\
Fetal heart rate (beats per minute) & 148 & 152 \\
Fundal height (cm) & 15 & 29 \\
PVT (per $\mu \mathrm{L})$ & 340 & 478 \\
PVG (per $\mu \mathrm{L})$ & 136 & 62 \\
Parasite clearance (days) & 1 & 2 \\
RBC $\left(\times 10^{6} / \mu \mathrm{L}\right)$ & $3.63(2.81-4.49)$ & $4.0(2.71-4.73)$ \\
HCT (\%) & $31.2^{*}(30-39)$ & $35.1(28.0-40.0)$ \\
HB (g/dL) & $10.0(9.7-14.8)$ & $11.9(9.5-15.0)$ \\
Reticulocytes $(\%)$ & $5.4(0.8-2.0)$ & $\mathrm{NA}$ \\
WBC $\left(\times 10^{3} / \mu \mathrm{L}\right)$ & $8.5(5.6-14.8)$ & $10.4(5.9-16.9)$ \\
Platelets $\left(\times 10^{3} / \mu \mathrm{L}\right)$ & $245(155-409)$ & $217(146-429)$ \\
\hline
\end{tabular}

Reference ranges are shown in brackets and were adjusted to the corresponding trimester [38]

$\mathrm{CHQ}$, Chloroquine; $\mathrm{COA}+$, augmented regimen of artemether-lumefantrine $(5$ tablets twice daily for 4 days); $\mathrm{EGA}$, estimated gestational age; $\mathrm{HB}$, haemoglobin; HCT, haematocrit; NA, not available; PVG, Plasmodium vivax gametocytes; PVT, Plasmodium vivax trophozoites; RBC, red blood cell count; WBC, white blood cell count

* $\mathrm{HCT}$ declined to $27 \%$ on day 5

following treatment with $\mathrm{COA}+$ as this was not in the trial protocol.

Parasitaemia cleared after 1 day of treatment and no fever was recorded on 6-hourly temperature measurements. The patient was discharged on day 5 . Since the HCT value on the day of discharge was only $27 \%$, the patient was prescribed anaemia treatment (ferrous sulfate $400 \mathrm{mg}$ twice daily and folic acid $5 \mathrm{mg}$ once daily). The patient followed ANC once weekly for 63 days as per study protocol. Malarial screening was repeatedly negative and the HCT rose to $33 \%$ after 2 weeks of anaemia treatment.

Nearly 5 months later, at an estimated gestational age of $35+4$ weeks, routine malaria screening by microscopy during the ANC visit detected $P$. vivax. She had no symptoms and was treated with CHQ $(10 \mathrm{mg} / \mathrm{kg}$ per day for 2 days and $5 \mathrm{mg} / \mathrm{kg}$ per day for 1 day) following the standard treatment recommended by the WHO [7]. Parasites cleared after 2 days of CHQ and the HCT (35\%) was normal. As with the first P. vivax episode, no prophylaxis was given.

\section{Delivery}

Routine symphysis fundal height measurement indicated poor fetal growth, which was confirmed by fetal anthropometry using ultrasound. After induction of labour for severe IUGR, a normal female infant was birthed in an SMRU clinic with an estimated gestational age of $38+6$ weeks. The Apgar scores were 9 and 10 at 1 and 5 min, respectively. On examination, small for gestational age $(<1$ st centile by international standards) was confirmed with a body weight of $1980 \mathrm{~g}$, a head circumference of $30 \mathrm{~cm}(<3 \mathrm{rd}$ centile) and a body length of $45 \mathrm{~cm}$ $(<10$ th centile) [26]. Maternal, neonatal, umbilical cord, and placental blood smears were negative for malaria parasites. No risk factors other than the $P$. vivax episodes during pregnancy were identified for the severe IUGR.

\section{Postpartum radical cure}

After delivery the mother complied with study followup visits and no more complications arose. At 5 months postpartum the young mother was planning to move to a remote region with limited access to adequate health care. Radical curative treatment with PMQ for $P$. vivax was recommended before she moved, given the risk of anaemia and poor outcome of a subsequent pregnancy, despite being 1 month short of the WHO recommendation at that time (2016) for lactating mothers of 6 months [7].

Before administration of PMQ, the mother and infant were tested for G6PD deficiency using a FST; both were confirmed phenotypically normal. At baseline, the mother's HCT was $41 \%$ [reticulocytes $3 / 1000$ red blood cells (RBCs)] and the infant's HCT was $36 \%$; both blood slides were negative for $P$. vivax and the physical examination was unremarkable.

Daily weight-based treatment with PMQ $(0.5 \mathrm{mg} / \mathrm{kg} /$ day) for 14 days total was prescribed; the 4 tablets daily translated into an actual dose of $0.52 \mathrm{mg} / \mathrm{kg} /$ day (weight $58 \mathrm{~kg}$ ). The first dose was supervised in the clinic. At follow-up on the 7th day none of the commonly observed PMQ side effects, such as abdominal pain, nausea or vomiting was reported [9] but the patient complained of dizziness. The HCT was $27.4 \%$ (reference range for nonpregnant adult female 35.4-44.4\%), an absolute reduction of $13.6 \%$ (from 41 to $27.4 \%$ ), and equivalent to fractional reduction of $33 \%$. Acute anaemia was confirmed with additional haematologic parameters and the patient was admitted for observation (Table 2).

Biochemistry showed normal kidney function and, apart from a mildly increased direct bilirubin $(0.84 \mathrm{mg} /$ 
Table 2 Blood results before, during, and after primaquine radical cure at 5 months postpartum

\begin{tabular}{|c|c|c|c|c|c|c|}
\hline & $\begin{array}{l}\text { Day } 1 \text { (before } \\
\text { PMQ) }\end{array}$ & Day 4 & Day 7 & Day $10-12$ & 11 weeks & 7 months \\
\hline НСТ (35.4-44.4\%) & $41 \%$ & - & $27.4 \%$ & $30 \%$ & $37 \%$ & $36.1 \%$ \\
\hline Reticulocytes (per 1000 RBC) & 3 & - & 40 & - & - & - \\
\hline $\mathrm{RBC}(4.00-5.20 \times 106 / \mu \mathrm{L})$ & - & - & 3.14 & - & - & - \\
\hline $\mathrm{Hb}(12.0-15.8 \mathrm{~g} / \mathrm{dL})$ & - & - & 8.9 & - & - & 11.9 \\
\hline G6PD FST & Normal & - & - & - & - & - \\
\hline $\begin{array}{l}\text { G6PD activity* (IU/gHb) [\% popula- } \\
\text { tion median] }\end{array}$ & - & - & 7.7 [103\%] & - & - & 4.62 [62\%] \\
\hline Infant $\mathrm{HCT}$ & $36 \%$ & $38 \%$ & $33 \%$ & $33 \%$ & - & $36 \%$ \\
\hline Infant G6PD FST & Normal & & - & - & - & - \\
\hline
\end{tabular}

* Activity measured by spectrophotometry

$\mathrm{dL}$ [normal range: 0.3-1.3]) and alkaline phosphatase (132 U/L [33-96]), normal liver function tests. At 236 U/L (115-221) lactate dehydrogenase was slightly elevated. The direct Coombs test was negative and there was no haemoglobinuria. Otherwise, history and physical examination were unremarkable.

Given the clinical picture, G6PD deficiency was suspected despite the normal G6PD FST result as the FST has poor sensitivity to detect intermediate G6PD activity levels. The G6PD activity was then quantified by spectrophotometry and was found to be normal at $7.7 \mathrm{IU} / \mathrm{gHb}$ (population median: $7.51 \mathrm{IU} / \mathrm{gHb}$ ) [27], suggesting a false normal result in a haemolytic state $(\mathrm{Hb} 8.9 \mathrm{mg} / \mathrm{dL})$.

The patient's body weight was rechecked and found to be $56.5 \mathrm{~kg}$. With no signs of severe haemolysis and no evidence of G6PD deficiency, the PMQ course was continued under supervision at a corrected dose of 3.5 tablets daily (actual dose was $0.46 \mathrm{mg} / \mathrm{kg} /$ day for the remaining 7 days of treatment). The treatment was well tolerated from this point onwards and the woman's clinical condition improved. To treat the anaemia, the patient was provided with a 28-day course of ferrous sulfate, folic acid and vitamin B12. Since the mother was still breastfeeding, the infant's HCT was also followed and remained relatively stable (day $0,4,7$ and 10 the HCT was 36,38 , 33 and $33 \%$, respectively, fractional reduction of $8 \%$ ). On day 12 of PMQ, the maternal HCT had increased to $30 \%$, symptoms had resolved, and the patient requested to be discharged home. 11 weeks later, the HCT was $37 \%$, and the patient was well. The child's vaccination schedule was completed, and the 6-month motor milestones were normal.

\section{Additional laboratory findings following radical cure} Approximately 1 year after delivery, the mother returned with the infant for follow-up as part of the trial described earlier. Since the previous quantification of the G6PD activity during the haemolytic episode was considered unreliable (due to increased reticulocyte count and low $\mathrm{Hb}$ level), the staff offered the woman to repeat quantitative G6PD testing while in steady state (HCT 36.1\%). This time the G6PD activity was found to be $4.62 \mathrm{IU} / \mathrm{gHb}$, which corresponds to $62 \%$ of the population mean and is highly suggestive of G6PD heterozygosity [28]. Following this new finding, the patient and infant were screened for the most common G6PD gene mutations seen in the area (Mahidol, Chinese-4, Viangchan, Mediterranean variants) [29]. The coding regions from exon 2 to exon 13 of the G6PD gene of the mother were then sequenced according to protocol from Kim et al. [30] but no mutation was found. Mutations in non-coding regions have been linked to deficient G6PD activity [31]; however, in this case no further investigations were pursued. Interestingly the female infant was found to be heterozygous for the Mahidol variant. CYP2D6 genotyping was not performed but could have helped to clarify the haemolytic pattern.

\section{Conclusion}

Recognizing gender-specific aspects of disease acquisition, detection, treatment options, and response to treatment can help ensure that health policies are effective and equitable. As the historical legacy of gender-based inequality is deeply embedded in medical research and the health sector, action to prevent undue gender-specific disparities in health outcomes is an ethical imperative and mandated in the SDG [32].

In this case report of a female experiencing $P$. vivax in pregnancy, multiple layers of a gender-specific morbidity occur (Fig. 1). The reproductive impact of malaria is limited to females, and they suffer a double burden of more severe personal illness and poor outcomes for their offspring. The negative effect of $P$. vivax infection, especially 


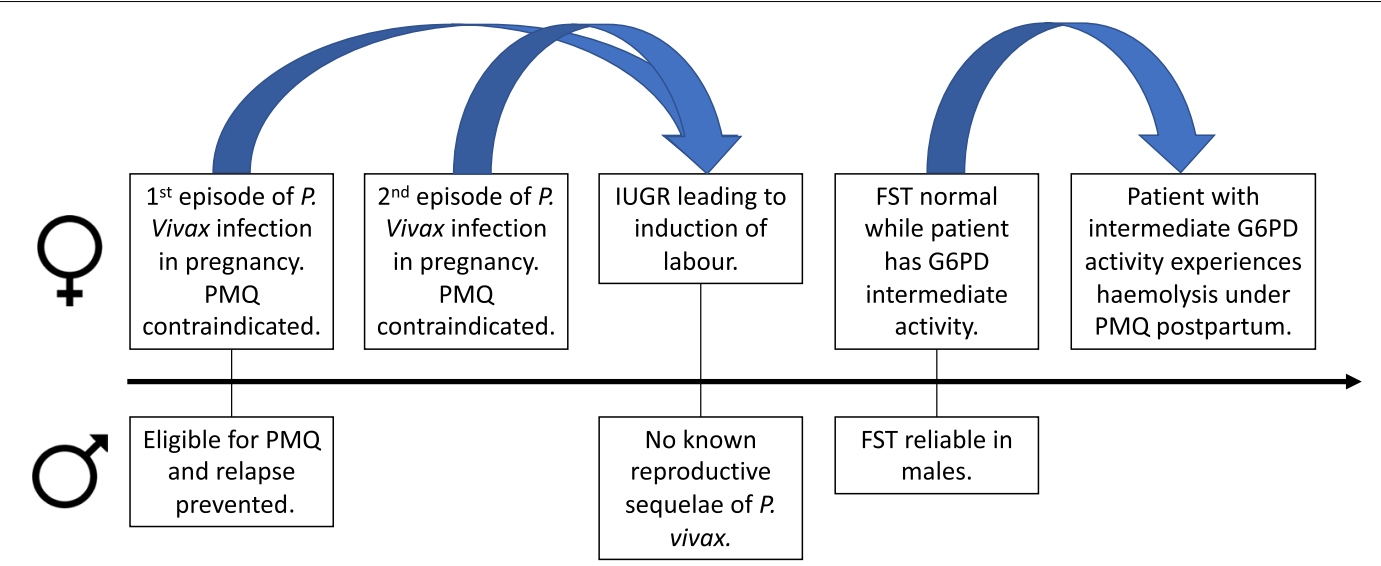

Fig. 1 Comparison of timeline of Plasmodium vivax infection in this pregnant woman to a hypothetical male patient with Plasmodium vivax

recurrent infections, on birth weight has been demonstrated, and this increases both short- and long-term morbidity and mortality for affected infants [5].

The second layer of a gender-specific morbidity results from the ineligibility of many females for radical cure due to their reproductive stage. It is estimated that about $13 \%$ of females with $P$. vivax are temporarily ineligible for radical cure due to pregnancy or lactation [6], and the proportion who eventually receive 8 -aminoquinolones postpartum as recommended by the WHO is unknown. Relapse is the main source of $P$. vivax infection in areas of low endemicity and contributes to chronic anaemia. A recent metanalysis confirms that, despite short term risk of haemolysis, radical cure with primaquine improves hematologic outcomes in the long term [33]. In most $P$. vivax-endemic regions, anaemia is a major public health problem, which contributes to maternal mortality [34]. The only strategies currently available to prevent harm from recurrent vivax malaria in pregnancy are weekly chemoprophylaxis with $\mathrm{CHQ}$ in pregnant women following their first episode of malaria, as recommended by the WHO, and early detection and treatment. Compelling theoretical concerns of iatrogenic fetal anaemia or hydrops prevent the use of 8-amnioquinolones in pregnancy, but there are no actual reports of adverse pregnancy outcomes after inadvertent administration. A recent publication included a report of 52 women in Brazil treated with primaquine during pregnancy (some repeatedly) without adverse birth outcomes [35]. However, G6PD testing was not done on the infants, and it is not unlikely that they were all G6PD normal. As other haemolytic drugs (eg. nitrofurantoin, dapsone) are used in pregnancy when indicated, it is conceivable that 8-aminoquinolones could be used as well. However, establishing the safety of current or new hypnozoiticidal drugs would require a cautious and systematic approach, starting with in silica or ex-vivo models of trans-placental transfer. Establishing safety during breastfeeding is considerably more straightforward. Pharmacokinetic data suggest that the 6-month postpartum delay for primaquine is unnecessarily long as PMQ is excreted into mature breast milk in negligible amounts [36]. Further information is needed on safety of PMQ in the neonatal period, and there is currently no data on TQ and lactation. Information on 8 -aminoquinolones in the neonatal period are urgently needed as the opportune moment for radical cure is post-partum before the woman leaves the birth facility. Our current institute policy is weekly chemoprophylaxis with $\mathrm{CHQ}$ until delivery after treatment of blood stages in women who experience a $P$. vivax infection, followed by radical cure with PMQ after 1 -month postpartum. Prior to discharge from the perinatal care unit, the patients are reminded of the significance of radical cure, and given a follow-up appointment.

The final layer of gender specific morbidity relates to tests for G6PD deficiency. When females are not pregnant or breastfeeding and are eligible for 8-aminoquinolones, they are at higher risk for misdiagnosis of G6PD deficiency by current qualitative testing [16]. This case involved a woman with only moderately decreased G6PD activity at $62 \%$ of the population mean, resulting in moderate but symptomatic haemolysis. It is expected that a fluorescent spot test (or an equivalent RDT) would classify heterozygous females with a G6PD activity as low as $30-40 \%$ as G6PD normal. In fact, Bancone et al. found that $60 \%$ of G6PD heterozygous females were misdiagnosed by qualitative screening [28]. Due to the loss of G6PD-deficient cells to haemolysis during illness, heterozygote females with even less than $30-40 \%$ activity could be misdiagnosed as normal if the test is done 
during an acute $P$. vivax episode. Such a misdiagnosis could result in fatal haemolysis if 8 -aminoquinolines are inappropriately prescribed to females with intermediate G6PD activity, which is an important consideration as the new single radical curative dose TQ has a long terminal half-life. Field-validated point-of-care quantitative G6PD tests are an urgent priority to meet the ambitious WHO target of reducing malaria by $90 \%$ by the year 2030 [37]. This is especially true in areas with high prevalence of G6PD deficiency in order to avoid iatrogenic morbidity and mortality for females. Testing should be offered when individuals are healthy, such as at a preconception visit at the community level or during a routine antenatal care visit.

In this case the mother received PMQ while she continued to breastfeed a female infant with a G6PD heterozygous genotype (Mahidol variant) without adverse effects on the infant. Given the pharmacokinetics of primaquine during lactation and the dose-dependent nature of primaquine-induced haemolysis, it is very unlikely that the clinically insignificant $8 \%$ fractional reduction in HCT seen in this infant is due to drug exposure via breastmilk. However, the fact that she was classified as G6PD normal by FST further highlights the need for improved G6PD testing modalities.

Improving care and prevention of malaria in pregnancy, reducing unnecessary exclusion of women from radical cure, and providing quantitative G6PD screening that is as accurate for females as it is for males are achievable steps towards the SDG for a more equitable and malaria-free world.

\begin{abstract}
Abbreviations
ALT: alanine aminotransferase; ANC: antenatal care; AST: aspartate aminotransferase; $\mathrm{CHQ}$ : chloroquine; $\mathrm{COA}+$ : augmented regimen of artemether-lumefantrine; EGA: estimated gestational age; FDA: food and drug administration; FST: fluorescent spot test; G6PD: glucose-6-phosphate dehydrogenase; HB: haemoglobin; HCT: haematocrit; IUGR: intra-uterine growth restriction; NA: not available; OD: once a day; PMQ: primaquine; PVG: Plasmodium vivax gametocytes; PVT: Plasmodium vivax trophozoites; RBC: red blood cell count; RDT: rapid diagnostic test; SDG: Sustainable Development Goals; SMRU: Shoklo Malaria Research Unit;TQ: Tafenoquine; WBC: white blood cell count; WHO: World Health Organization.
\end{abstract}

\section{Acknowledgements}

The Shoklo Malaria Research Unit is part of the Wellcome Trust Mahidol University Oxford Tropical Medicine Research Programme supported by the Wellcome Trust of Great Britain (Major Overseas Programme-Thailand Unit Core Gran: WT-106698).

\section{Authors' contributions}

Conceptualization: TB, MEG, RM. Data curation: TB, MP, GG, GB. Formal analysis: TB, MEG, RM, CSC, GB. Investigation: GG, GB. Methodology: CSC, GB. Supervision: FN, RM. Validation: CSC, GB, KC, FN, RM. Writing-original draft: TB, MEG, RM. Writing - reviewing \& editing: TB, MEG, GG, CSC, GB, MP, KC, FN, RM. The contributor's roles listed above follow the Contributor Roles Taxonomy (CRediT) managed by The Consortia Advancing Standards in Research Administration Information (CASRAI) (https://casrai.org/credit/). All authors read and approved the final manuscript.

\section{Funding}

No specific grant from any funding agency in the public, commercial, or notfor-profit sectors was received in preparation of this case report.

\section{Availability of data and materials}

Not applicable.

\section{Ethics approval and consent to participate}

Not applicable.

\section{Consent for publication}

Written informed consent was obtained from the patient for publication of this case report. A copy of the written consent is available for review by the Editor-in-Chief of this journal.

\section{Competing interests}

The authors declare that they have no competing interests.

\section{Author details}

1 Shoklo Malaria Research Unit, Mahidol-Oxford Tropical Medicine Research Unit, Faculty of Tropical Medicine, Mahidol University, P.O. Box 46, 68/31 Bann Tung Road, Mae Sot 63110, Thailand. ${ }^{2}$ Swiss Tropical and Public Health Institute, Basel, Switzerland. ${ }^{3}$ University of Basel, Basel, Switzerland. ${ }^{4}$ Centre for Tropical Medicine and Global Health, Nuffield Department of Medicine, University of Oxford, Old Road Campus, Oxford, UK. ${ }^{5}$ Mahidol-Oxford Tropical Medicine Research Unit, Faculty of Tropical Medicine, Mahidol University, Bangkok, Thailand.

Received: 17 December 2019 Accepted: 13 January 2020 Published online: 22 January 2020

\section{References}

1. Diiro GM, Affognon HD, Muriithi BW, Wanja SK, Mbogo C, Mutero C. The role of gender on malaria preventive behaviour among rural households in Kenya. Malar J. 2016;15:14.

2. Braveman P, Gruskin S. Defining equity in health. J Epidemiol Community Health. 2003;57:254-8.

3. WHO. World malaria report 2018. Geneva: World Health Organization, 2018. https://apps.who.int/iris/handle/10665/275867.

4. Moore KA, Fowkes FJI, Wiladphaingern J, Wai NS, Paw MK, Pimanpanarak $M$, et al. Mediation of the effect of malaria in pregnancy on stillbirth and neonatal death in an area of low transmission: observational data analysis. BMC Med. 2017;15:98.

5. Moore KA, Simpson JA, Wiladphaingern J, Min AM, Pimanpanarak M, Paw MK, et al. Influence of the number and timing of malaria episodes during pregnancy on prematurity and small-for-gestational-age in an area of low transmission. BMC Med. 2017; 15:117.

6. Watson J, Taylor WRJ, Bancone G, Chu CS, Jittamala P, White NJ. Implications of current therapeutic restrictions for primaquine and tafenoquine in the radical cure of vivax malaria. PLoS Negl Trop Dis. 2018;12:e0006440.

7. WHO. Guidelines for the treatment of malaria. 3rd Edn. Geneva: World Health Organization, 2015. http://www.ncbi.nlm.nih.gov/books/NBK29 4440/.

8. Watson J, Taylor WR, Menard D, Kheng S, White NJ. Modelling primaquine-induced haemolysis in G6PD deficiency. ELife. 2017;6:e23061.

9. Recht J, Ashley EA, White NJ. Safety of 8-aminoquinoline antimalarial medicines. Geneva: World Health Organization, 2014. http://www.who. int/malaria/publications/atoz/9789241506977/en/.

10. Ashley EA, Recht J, White NJ. Primaquine: the risks and the benefits. Malar J. 2014;13:418.

11. Chu CS, Bancone G, Moore KA, Win HH, Thitipanawan N, Po C, et al. Haemolysis in G6PD heterozygous females treated with primaquine for Plasmodium vivax malaria: a nested cohort in a trial of radical curative regimens. PLoS Med. 2017;14:e1002224.

12. Takeuchi R, Lawpoolsri S, Imwong M, Kobayashi J, Kaewkungwal J, Pukrittayakamee S, et al. Directly-observed therapy (DOT) for the radical 14-day primaquine treatment of Plasmodium vivax malaria on the Thai-Myanmar border. Malar J. 2010;9:308. 
13. White NJ. Tafenoquine-a radical improvement? N Engl J Med. 2019;380:285-6.

14. Beutler E, Yeh M, Fairbanks VF. The normal human female as a mosaic of X-chromosome activity: studies using the gene for G-6-PD-deficiency as a marker. Proc Natl Acad Sci USA. 1962;48:9-16.

15. Ley B, Winasti Satyagraha A, Rahmat H, von Fricken ME, Douglas NM, Pfeffer DA, et al. Performance of the Access Bio/CareStart rapid diagnostic test for the detection of glucose-6-phosphate dehydrogenase deficiency: a systematic review and meta-analysis. PLoS Med. 2019;16:e1002992.

16. Domingo GJ, Advani N, Satyagraha AW, Sibley CH, Rowley E, Kalnoky M, et al. Addressing the gender-knowledge gap in glucose-6-phosphate dehydrogenase deficiency: challenges and opportunities. Int Health. 2019;11:7-14

17. Luzzatto L, Seneca E. G6PD deficiency: a classic example of pharmacogenetics with on-going clinical implications. Br J Haematol. 2014;164:469-80.

18. Domingo GJ, Satyagraha AW, Anvikar A, Baird K, Bancone G, Bansil P, et al. G6PD testing in support of treatment and elimination of malaria: recommendations for evaluation of G6PD tests. Malar J. 2013;12:391.

19. Bancone G, Gornsawun G, Chu CS, Porn P, Pal S, Bansil P, et al. Validation of the quantitative point-of-care CareStart biosensor for assessment of G6PD activity in venous blood. PLoS ONE. 2018;13:e0196716.

20. Alam MS, Kibria MG, Jahan N, Price RN, Ley B. Spectrophotometry assays to determine G6PD activity from Trinity Biotech and Pointe Scientific G6PD show good correlation. BMC Res Notes. 2018;11:855.

21. WHO Malaria Policy Advisory Committee and Secretariat. Malaria Policy Advisory Committee to the WHO: conclusions and recommendations of eighth biannual meeting (September 2015). Malar J. 2016;15:117.

22. Boel ME, Rijken MJ, Leenstra T, Phyo AP, Pimanpanarak M, Keereecharoen $\mathrm{NL}$, et al. Malaria in the post-partum period; a prospective cohort study. PLOS ONE. 2013:8:e57890.

23. Taylor AR, Watson JA, Chu CS, Puaprasert K, Duanguppama J, Day NPJ, et al. Resolving the cause of recurrent Plasmodium vivax malaria probabilistically. Nat Commun. 2019;10:1-11.

24. Chu CS, Phyo AP, Lwin KM, Win HH, San T, Aung AA, et al. Comparison of the cumulative efficacy and safety of qhloroquine, artesunate, and chloroquine-primaquine in Plasmodium vivax malaria. Clin Infect Dis. 2018:67:1543-9.

25. Nosten F, McGready R, Simpson JA, Thwai KL, Balkan S, Cho T, et al. Effects of Plasmodium vivax malaria in pregnancy. Lancet. 1999;354:546-9.

26. Villar J, Cheikh Ismail L, Victora CG, Ohuma EO, Bertino E, Altman DG, et al. International standards for newborn weight, length, and head circumference by gestational age and sex: the Newborn Cross-Sectional Study of the Intergrowth-21st Project. Lancet. 2014;384:857-68.

27. Bancone G, Chu CS, Chowwiwat N, Somsakchaicharoen R, Wilaisrisak $P$, Charunwatthana $P$, et al. Suitability of capillary blood for quantitative assessment of G6PD activity and performances of G6PD point-of-care tests. Am J Trop Med Hyg. 2015;92:818-24.
28. Bancone G, Gilder ME, Chowwiwat N, Gornsawun G, Win E, Cho WW, et al. Prevalences of inherited red blood cell disorders in pregnant women of different ethnicities living along the Thailand-Myanmar border. Wellcome Open Res. 2017;2:72.

29. Bancone G, Chu CS, Somsakchaicharoen R, Chowwiwat N, Parker DM, Charunwatthana P, et al. Characterization of G6PD genotypes and phenotypes on the northwestern Thailand-Myanmar border. PLoS ONE. 2014;9:e116063.

30. Kim S, Nguon C, Guillard B, Duong S, Chy S, Sum S, et al. Performance of the CareStart G6PD deficiency screening test, a point-of-care diagnostic for primaquine therapy screening. PLOS ONE. 2011;6:e28357.

31. Efferth T, Bachli EB, Schwarzl SM, Goede JS, West C, Smith JC, et al. Glucose-6-phosphate dehydrogenase (G6PD) deficiency-type Zurich: a splice site mutation as an uncommon mechanism producing enzyme deficiency. Blood. 2004;104:2608.

32. Gupta GR, Oomman N, Grown C, Conn K, Hawkes S, Shawar YR, et al. Gender equality and gender norms: framing the opportunities for health. Lancet. 2019;393:2550-62.

33. Commons RJ, Simpson JA, Thriemer K, Chu CS, Douglas NM, Abreha T, et al. The haematological consequences of Plasmodium vivax malaria after chloroquine treatment with and without primaquine: a WorldWide Antimalarial Resistance Network systematic review and individual patient data meta-analysis. BMC Med. 2019;17:151.

34. McGready R, Boel M, Rijken MJ, Ashley EA, Cho T, Moo O, et al. Effect of early detection and treatment on malaria related maternal mortality on the north-western border of Thailand 1986-2010. PLOS ONE. 2012;7:e40244.

35. Pincelli A, Neves PAR, Lourenço BH, Corder RM, Malta MB, Sampaio-Silva J, et al. The hidden burden of Plasmodium vivax malaria in pregnancy in the Amazon: an observational study in Northwestern Brazil. Am J Trop Med Hyg 2018;99(1):73-83

36. Gilder ME, Hanpithakphong W, Hoglund RM, Tarning J, Win HH, Hilda N, et al. Primaquine pharmacokinetics in lactating women and breastfed infant exposures. Clin Infect Dis. 2018;67:1000-7.

37. WHO. Global technical strategy for malaria 2016-2030. Geneva:World Health Organization, 2015. http://www.who.int/malaria/areas/globa I_technical_strategy/en/.

38. Abbassi-Ghanavati M, Greer LG, Cunningham FG. Pregnancy and laboratory studies: a reference table for clinicians. Obstet Gynecol. 2009;114:1326-31.

\section{Publisher's Note}

Springer Nature remains neutral with regard to jurisdictional claims in published maps and institutional affiliations.

Ready to submit your research? Choose BMC and benefit from:

- fast, convenient online submission

- thorough peer review by experienced researchers in your field

- rapid publication on acceptance

- support for research data, including large and complex data types

- gold Open Access which fosters wider collaboration and increased citations

- maximum visibility for your research: over 100M website views per year

At BMC, research is always in progress.

Learn more biomedcentral.com/submissions 